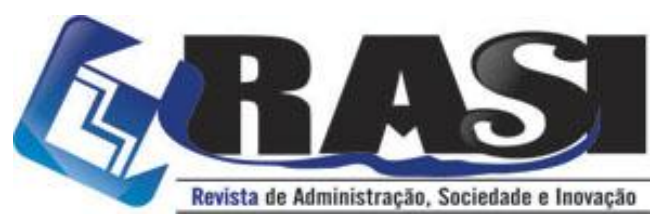

http://www.rasi.uff.br

RASI, Volta Redonda/RJ, v. 4, n. 2, pp. 127-139, jul./dez. 2018

\title{
Gestão de Redes de Empresas e Technology Roadmapping: possibilidades para uma abordagem estratégica
}

\author{
Luiz Guilherme Rodrigues Antunes (UFLA) - luguiantunes@yahoo.com.br \\ Thais Assis de Souza (UFLA) - assis.sthais@gmail.com \\ Cléber Carvalho de Castro (UFLA) - clebercastro@dae.ufla.br
}

\begin{abstract}
RESUMO: Como uma estrutura complexa de interações, as redes interorganizacionais devem ser gerenciadas estrategicamente de forma a controlar variáveis dos negócios. Como uma ferramenta de apoio ao planejamento estratégico, o technology roadmapping (TRM), a partir de estruturação de roteiros baseados em tempo, objetivos e atividades setoriais, capacita a gestão a reconhecer especificidades e traçar metas que alinhem objetivos estratégicos à disponibilidade tecnológica. Porém, como o technology roadmap pode ser associado com a gestão das redes de empresas? Como esta ferramenta pode ser utilizada a favor dessas configurações? Essas perguntas norteiam este ensaio teórico. Afim de responde-las, definiu-se como objetivo do presente artigo evidenciar a relação entre redes de empresa e technology roadmapping de forma a apresentar conciliações que facilitem e estimulem novos trabalhos no campo com foco em planejamento estratégico de redes interorganizacionais. Com intuito de complementar as evidências teóricas, também foi desenvolvido uma revisão de escopo sobre as temáticas afim de ressaltar a importância da aplicação do instrumento neste contexto. Como resultado, verificouse que o TRM apoia a gestão estratégica de redes no sentido em que fundamenta processos estratégicos, tais como: criação de portfólio de serviços e produtos, transferência de tecnologia e de conhecimento, integração de competências, dentre outros.
\end{abstract}

PALAVRAS-CHAVE: Redes interorganizacionais. Technology Roadmapping. Gestão de Redes.

\section{Management of Business Networks and Technology Roadmapping: possibilities for a strategic approach}

ABSTRACT: Presenting itself as a complex structure of interactions, interorganizational networks must be managed strategically in order to control business variables that influence performance. As a tool to support strategic planning, technology roadmapping (TRM), based on structuring time-based roadmaps, objectives and division of activities by sectoral lines of action, enables management to recognize specificities and set goals that align strategic objectives technological availability. However, how can technology roadmap be associated with the management of corporate networks? How can this tool be used in favor of these settings? These questions guide this theoretical essay. In order to respond, it was defined as the goal of this article to highlight the relationship between company networks and technology roadmapping in order to present conciliations that facilitate and stimulate new work in the field focusing on strategic planning of interorganizational networks. In order to complement the theoretical evidence, a scope review was also developed to highlight the importance of applying the instrument in this context. As a result, it was verified that TRM can support the network's management in order to finance the strategic processes, such as: creation of services and products' portfolio, transfer of technology and knowledge, integration of skills, among others.

KEYWORDS: Interorganizational networks. Technology Roadmapping. Network Management.

\section{Universidade \\ U. Federal Fluminense}

R. Desembargador Ellis Hermydio Figueira, 783, Bloco A, sl. 218, Aterrado. 27213-415 - Volta Redonda, RJ - Brasil www.uff.br

Copyright (C) 2018 RASI. Todos os direitos, até mesmo de tradução, são reservados. É permitido citar parte de artigos sem autorização prévia, desde que seja identificada a fonte 


\section{Gestão de Redes de Empresas e Technology Roadmapping: possibilidades para uma abordagem estratégica}

\section{Introdução}

A gestão de redes tem atenção principal sobre atores e os processos de interação existentes (Fleury \& Teixeira, 2011), porém, para atingir objetivos específicos é preciso identificar oportunidades e selecionar, dentre as opções, aquela que, a partir de uma visão estratégica, é a mais viável a ser selecionada (Fleury, 2014). Segundo Fleury (2014), definir ações planejadas em agenda vai além de ações de comunicação, pois envolve instrumentos que visam aprendizagem, que facilitem processos de avaliação, de mobilização e de coordenação de ações interdependentes. A autora ainda afirma que "a literatura de redes mostra que esse não é um processo fácil, pois exige a compatibilização de diferentes culturas e processos em conjunto e colaborativo, que requer um andamento similar" (Fleury, 2014, p. $11)$.

No contexto de estratégia, considerando o grau de interação com o ambiente, o comportamento assumido frente ao mercado e às diferentes variáveis ambientais influenciadoras levam a empresa ao sucesso adquirido (Alday, 2000). No contexto de redes, tais variáveis ganham maiores proporções e necessitam, então, de maiores controles. Surge, portanto, o planejamento estratégico como processo gerencial que visa inserir a organização e sua missão no ambiente no qual atuam. O estabelecimento de direção minimiza deficiências e maximiza resultados a partir de uma maior e melhor interação com o ambiente (Alday, 2000; Chiavenato \& Sapiro, 2003).

Como um dos métodos de apoio à gestão estratégica o technology roadmapping (TRM) apresenta-se como ferramenta que favorece a comunicação entre atores a partir de estruturação de roteiros baseado em tempo, objetivos e divisão de atividades por linhas setoriais de ação (Lee \& Park, 2005; Phaal, et al., 2005). Com característica de flexibilidade quanto à aplicação em diferentes contextos e sob diferentes objetivos, além de possibilitar adequação customizada na sua arquitetura, conteúdo, tempo e desenvolvimento do processo, o TRM trabalha a partir de três questões básicas: para onde estamos caminhando? onde nos encontramos atualmente? e o que fazer para chegar lá? (Lee \& Park, 2005; Phaal et al., 2001, 2005).

Frente a tais características do TRM, surgem os seguintes questionamentos como orientadores deste ensaio teórico: como o Technology Roadmapping pode ser associado com a gestão das redes de empresas? Como ela pode ser utilizada a favor dessas configurações? Afim de responde-las, definiu-se como objetivo do presente artigo evidenciar a relação entre redes de empresa, estas como novo formato organizacional, e technology roadmapping de forma a apresentar conciliações que facilitem e estimulem novos trabalhos no campo com foco em planejamento estratégico de redes interorganizacionais.

Advoga-se, portanto, que o TRM, como ferramenta de apoio estratégico, pode ser utilizada eficientemente nas redes de empresa de forma a auxiliar nas questões estratégicas de mercado, produto, tecnologia e demais aspectos, além de se caracterizar como adaptável à configuração da rede. Assim, defende-se a tese de que por meio da TRM é possível estabelecer uma gestão mais proativa da rede, enfocando os recursos, as redes de contatos, lideranças, stakeholders, negócios e inovação.

Essa pesquisa torna-se relevante à medida que se constata na literatura que o uso do TRM se encontra demasiadamente presente (Battistella et al., 2015). Porém, quando associados a redes de empresas, a literatura ainda é incipiente, sendo, portanto, uma lacuna de investigação. Em 
complemento, Kappel (2001) afirma que os sistemas complexos (como as redes interorganizacionais) representam uma estrutura que necessita fortemente de definições de roteiros distribuídos.

\section{Referencial Teórico}

\subsection{Redes de Empresas}

Ao final do século XX um conjunto de modificações ambientais abalaram a estabilidade, conservação, constância e equilíbrio do capitalismo gerencial, bem como as estruturas hierárquicas e burocráticas (Balestrin \& Verschoore, 2016). Intitulado de nova competição, o ambiente apresentou-se mais flexível devido à adaptabilidade das fronteiras organizacionais e pela busca constante de inovações (Balestrin \& Verschoore, 2016). Desse novo cenário, surgiu, na década de 1980, o termo "coopetição" que refere-se às relações interorganizacionais que envolvem aspectos de cooperação e competição (Lacoste, 2012). Neste sentido, a emergência de estratégias "coopetitivas" se mostram fundamentais para melhores resultados, sobrevivência das empresas no mercado (Merofa \& Bueno, 2009), e confirmaram que as organizações não se encontravam isoladas, mas inseridas em toda uma rede de relacionamentos (Prahalad \& Ramaswamy, 2004; Balestrin \& Verchoore, 2016).

Segundo Nohria (1992), um novo modelo de organização emergiu desse novo paradigma: as redes de empresas. Miles e Snow (1992) salientam que o movimento em direção às redes tornou-se mais evidente na mesma década, quando a concorrência internacional e as rápidas mudanças tecnológicas forçaram uma reestruturação através das indústrias e empresas. Para Nohria (1992), esse novo formato permitiu interligações laterais e horizontais dentro e entre empresas. Conforme Castells (2005), as redes são estruturas formais e com sistema de nós interligados. Os nós são os pontos de encontro entre atores, o que permite o fluxo de recursos entre os mesmos. Na organização em redes, os mesmos operam ao longo de vários processos que se reforçam, e podem apresentar-se desde grandes empresas que se descentralizam ou formam redes de parcerias, a pequenas e médias empresas que formam redes de negócio entre sí ou atuam como fornecedoras de grandes indústrias (Castells, 2005). Balestrin e Vargas (2004) apontam que as redes também são regidas por interações e interdependência entre as organizações, bem como cooperação e comprometimento. Segundo Johnson-Cramer, et al. (2007) citados por Balestrin e Verschoore (2014) o propósito das redes está relacionado com:

"Reunir atributos em uma estrutura dinâmica, sustentada por ações uniformizadas e descentralizadas que possibilitem uma adequação a um ambiente competitivo, em geral, marcado por rápidas transformações, pelo sucessivo crescimento das expectativas dos consumidores e pela contínua busca pela eficiência (JohnsonCrameret al., 2007, p.527)".

Já Grandori e Soda (1995) afirmam que as redes são modos de organização das atividades econômicas por meio da coordenação e cooperação entre empresas. Em outras palavras, são conjuntos de mecanismos de integração que abarcam toda a gama de instrumentos de coordenação, desde comunicação informal a sistemas de informação e planejamento da rede, ocasionando em uma complexa estrutura de integração que complementa ou substitui os mecanismos de mercado. 
A coordenação está diretamente vinculada à gestão da rede. Assim, a cooperação entre os membros da rede não está associada apenas à gestão desses membros, mas à gestão da rede como um todo (Human \& Provan, 1997) e na gestão das ações coletivas como peças-chave para um desempenho superior (Balestrin \& Verschoore, 2016).

Para Balestrin e Verschoore (2016, p.148), "as redes de cooperação são organizações complexas e, como tais, precisam estar alicerçadas num modelo de gestão que possibilite sua sobrevivência e crescimento". Assim, os autores salientam que, por possuir características peculiares, as redes exigem gestores capacitados e instrumentos específicos de gestão. Outra contribuição importante é de Bortolaso, et al. (2013) que argumentam que na literatura sobre redes há diversos tópicos abordados como sendo características distintivas da gestão dessa forma organizativa. Assim, ressalta-se que as estratégias devem ser estabelecidas de forma clara e coletiva afim de se alcançar os objetivos comuns do grupo. Ademais, a condução do processo da estratégia deve ser conciliada entre todos os associados, devido ao fato que os gestores, as equipes de trabalho e os atores envolvidos necessitam trabalhar de maneira alinhada tentando progredir com a rede.

Por fim, no que concerne à inovação, "as parcerias interorganizacionais têm cada vez mais se tornado um componente central na estratégia corporativa" (Oliveira et al., 2012, p. 06). Dessa forma, as alianças entre empresas são processos de criação de valor conjuntas (Zajac \& Oslen, 1993) e sua gestão ao longo do tempo ocasiona distintas capacidades relacionais, sendo uma delas a inovação (Capaldo, 2007). Para Oliveira et al. (2012) as redes têm se tornado lócus da inovação, uma vez que são caracterizadas pela geração e teste de novas ideias em contexto flexível e colaborativo, bem como são ambientes de divisão dos custos e recursos, além de redutores de riscos a decisões erradas sobre tecnologia (Castells, 2007).

\subsection{Technology Roadmapping}

Technology roadmapping (TRM) é considerado na literatura como ferramenta, técnica, processo e metodologia (Phaal et al., 2004; Bernal et al., 2009; Santos et al., 2010). Todas essas nomenclaturas direcionam ao entendimento de utilização do TRM como um dos meios de apoio à gestão estratégica mais utilizados no alinhamento de tecnologias aos objetivos organizacionais (Lee \& Park, 2005; Phaal et al., 2005).

Com origem na abordagem adotada pela Motorola no final dos anos 1970, o TRM tem sido adotado e adaptado por diferentes organizações e setores que buscam a articulação de tecnologia com planos estratégicos, alinhando oportunidades de mercado ao desenvolvimento de produtos. A crescente popularidade é resultado proveniente, principalmente, da comunicação favorecida a partir da estruturação de roteiros, facilitando, assim, uma maior inclusão de atores, uma vez que o entendimento se torna comum em todos os níveis da fronteira organizacional (Phaal et al., 2001, 2005). A partir da utilização do TRM, a empresa angaria um status tecnológico a ser mantido e/ou melhorado de acordo com as metas estabelecidas (Bernal et al., 2009).

O formato do TRM tem características particulares representadas por meio de esquema de quadro baseado em cronologia e dividido em camadas que representam links tecnológicos de tomadas estratégicas. Para customização do TRM, dessa forma, devem ser contempladas necessidades específicas, setor, contexto, foco, pontos críticos, condicionantes, alternativas, informações disponíveis e demais aspectos organizacionais que irão direcionar o estágio de planejamento. Em geral, são mapeados o mercado, o produto e a tecnologia, porém, 
outras chaves podem ser incorporadas de acordo com a realidade apresentada (Phaal et al., 2001; Lee \& Park, 2005).

De forma a direcionar o desenvolvimento do TRM considerando estes desafios, Phaal et al. (2001) desenvolveram um guia de customização baseado em workshops com objetivos específicos que resultam na construção do relatório final. Este guia - T-Plan - foi resultante de pesquisa de três anos patrocinada por um conselho de pesquisa do Reino Unido. Os benefícios citados incluem identificação de gaps, redução de risco, direcionamento de esforços, participação multidisciplinar e desenvolvimento de linguagem comum.

Com o objetivo principal de certificar a alocação correta de capacidades no lugar e no momento certo de forma a atingir objetivos esperados, o TRM ainda tem sido muito referenciado à grandes corporações, apresentando-se como black box para empresas que não se encaixam nesse perfil, o que traz à tona a necessidade de criar caminhos para que o método seja utilizado por outras realidades de organizações, favorecendo o desenvolvimento destas e do mercado como um todo (Lee \& Park, 2005; Battistella et al., 2015).

Considerar especificidades para delimitar os limites e camadas do TRM é fundamental para que as demais atividades sejam orientadas e desenvolvidas de maneira adequada. Com as devidas considerações, será, então, possível o mapeamento e integração de atividades de forma a permitir que informações possam fluir considerando o fator tempo (Phaal et al., 2001). A Figura 01 apresenta uma tratativa integrativa de atividades com questionamento de limites abordados para delimitação do processo de roadmapping.

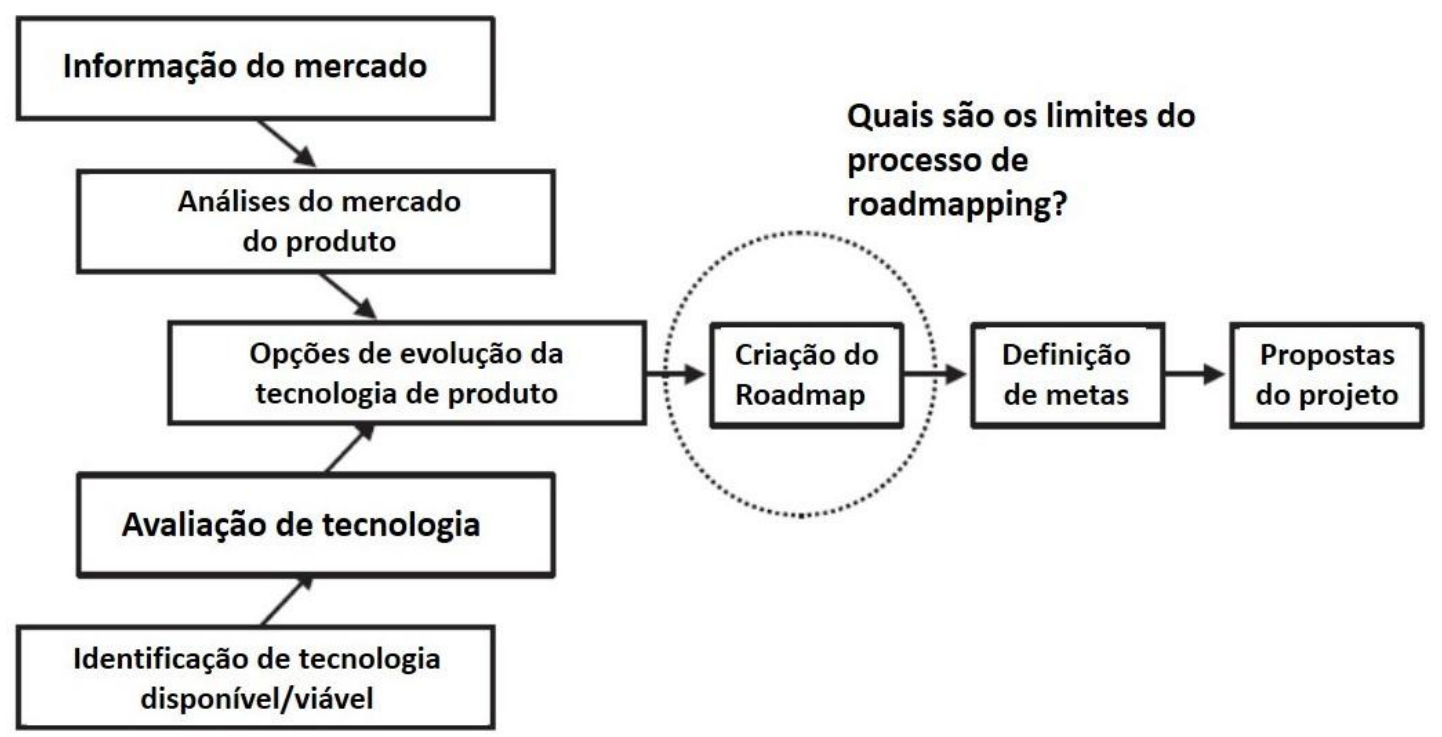

Figura 01: Entendendo os contornos do roadmapping

Fonte: EIRMA (1997), Technology roadmapping: delivering business vision. Working Group Report, 52, Paris, citado por Phaal, R., Farrukh, C.J.P. and Probert, D.R. (2001), T-Plan: the faststart to technology roadmapping planning your route to success, Institute for Manufacturing, University of Cambridge.

\section{Redes de Empresas e Technology Roadmapping: Tentativa de Conciliação}

A fim de ressaltar a relevância do estudo e atingir o objetivo deste ensaio teórico, decidiu-se elaborar uma revisão de escopo da temática de redes de empresas associada ao technology roadmapping, evidenciando na literatura as contribuições já existentes. 
Primeiramente, uma revisão de escopo, segundo Arksey e O'malley (2005), tem o objetivo de mapear rapidamente os principais conceitos que estruturam determinada temática, bem como suas principais fontes e tipos de evidências disponíveis. Sua realização objetiva examinar o alcance e a natureza da investigação em particular ou referir-se como uma própria técnica para publicação ou para identificar lacunas na literatura (Arksey \& O'malley, 2005; Rumrill et al., 2010). Assim, com a junção da revisão de escopo com o ensaio teórico é possível englobar essas duas finalidades.

Com o mapeamento metodológico seguiu-se os cinco passos propostos por Arksey e O’malley (2005) que são identificadas na Figura 02.

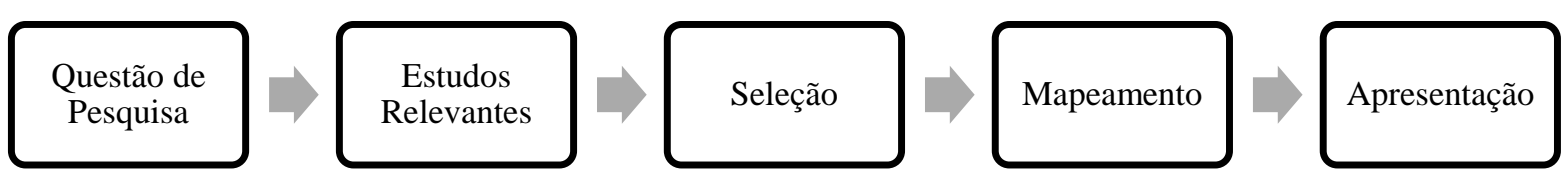

Figura 02. Etapas da Revisão de Escopo

A primeira etapa contemplou a definição do problema de pesquisa, que concentrou-se na pergunta: $O$ que já foi produzido na literatura sobre a relação entre tecnologia roadmapping e redes de empresas? Na segunda fase, estabeleceu-se onde e quais trabalhos seriam examinados na área a ser investigada. Para isso, foi escolhido a base da dados da Web of Science. Nesta base de dados, a coleta de artigos estabeleceu-se pelo campo tópico, com base em palavras-chaves presente em títulos, resumos, palavras-chaves de autor e keywords plus ${ }^{\circledR}$. O período de tempo estipulado para análise foi o padrão utilizado pela própria base de dados, de 1945 até 2016. A realização da busca se deu pelo cruzamento de palavras-chaves de redes de empresa (inter-firm, network organization, social network, interorganizational relationship, cluster, industrial district, aliance, cooperation network, network e milieu inovateur) e technology roadmapping (technology roadmap) com uso de caractere de truncagem (*). Utilizou-se também refinamentos para a seleção de amostras e, são elas: apenas registros classificados como "artigos"; em inglês; com área de pesquisa em "business economics" e "public administration"; e exclusão dos títulos de conferência. Dessa forma, foram obtidos como amostra 25 artigos relacionados com a temática pretendida. Na posterior etapa, a estratégia de seleção dos conteúdos pautou-se nos critérios de exclusão como disponibilidade dos artigos e leitura dos resumos, este último com a finalidade de analisar o alinhamento como o escopo da pesquisa. Obteve-se, portanto, três artigos referentes ao objeto de estudo.Na quarta etapa, sintetizou-se os dados qualitativos com a ajuda do software Excelß da Microsoft. A organização da planilha contou com a coleta de dados de ano de publicação, título do artigo, autores, relação com a temática, revista de publicação, objetivo, utilização do TRM, metodologia e contribuições. Por fim, a última etapa contempla a interpretação e análise dos dados, bem como a visão geral de todo o material avaliado (aqui apresentados).

Com base nesta metodologia, pode-se verificar que a relação entre os temas technology roadmapping e redes de empresas ainda é pouco explorada na literatura. Assim constatou-se que somente três artigos fazem algum tipo de relação entre essas temáticas. Esses artigos foram publicados recentemente, nos anos de 2012, 2013 e 2015, conforme aponta a Tabela 02: 
Tabela 02.

Artigos que associam redes de empresas com tecnologia roadmap.

\begin{tabular}{clcc} 
Ano & \multicolumn{1}{c}{ Título } & Autor (es) & Revista \\
\hline \multirow{2}{*}{2012} & $\begin{array}{l}\text { Maneuvering between networks to lead } \\
\text { A longitudinal case study in the } \\
\text { Semiconductor Industry }\end{array}$ & $\begin{array}{c}\text { Muller-Seitz e Sydow } \\
(2012)\end{array}$ & Long Range Planning \\
\hline \multirow{2}{*}{2013} & $\begin{array}{l}\text { Financing innovations in uncertain } \\
\text { networks - Filling in roadmap gaps in } \\
\text { the semiconductor industry }\end{array}$ & Lange et al. (2013) & Research Policy \\
\hline \multirow{2}{*}{2015} & $\begin{array}{l}\text { The extended map methodology: } \\
\text { Tecnhology roadmapping for SMEs } \\
\text { clusters }\end{array}$ & $\begin{array}{c}\text { Battistella et al. } \\
(2015)\end{array}$ & $\begin{array}{c}\text { Journal of } \\
\text { Engineering and } \\
\text { Technology } \\
\text { Managment }\end{array}$
\end{tabular}

Partindo-se dos objetivos das pesquisas acima mencionadas, o artigo de Muller-Seitz e Sydow (2012) buscou apresentar como e em que circunstâncias poderia uma organização liderar uma rede hierárquica. Desse modo, os autores utilizaram o technology roadmapping como uma ferramenta que forneceu um direcionamento para uma organização liderar a rede. $\mathrm{O}$ artigo de Lange et al. (2013) buscou explorar como os recursos financeiros dentro das redes de inovação são mobilizados e alocados. O TRM também foi utilizado como uma ferramenta que permitiu aos atores identificarem quais eram os seus componentes críticos, sobretudo aqueles que ainda precisavam ser desenvolvidos. Por fim, o último artigo, de Battistella et al. (2015), propôs uma metodologia específica para o TRM focado no contexto das pequenas e médias empresas.

No que tange aos autores que mais publicaram, destacam-se Muller-Seitz and Sydow com a publicação de dois trabalhos. Para finalizar as análises, os três artigos levantados pela revisão são empíricos e qualitativos. Os trabalhos de 2012 e 2013 foram elaborados com base em um estudo de caso, enquanto o de 2015 foi desenvolvido por uma pesquisa-ação.

A partir da revisão de escopo foi possível traçar outras integrações, conforme são apresentados nos parágrafos posteriores. No entanto, não é objetivo desta revisão esgotar as possibilidades de união das temáticas, mas apresentar mais oportunidades desta compatibilização. Cabe, portanto, aos pesquisadores e gerentes interessados a elaboração de trabalhos ou pesquisas contemplando esses e outros aspectos abordados neste ensaio teórico.

Partindo das contribuições de Phaal et al. (2001) e Lee and Park (2005), o technology roadmapping pode ser utilizado para mapeamento de mercado, produtos e tecnologias. No entanto, essa ferramenta deve ser utilizada de forma estratégica pela gestão da rede de forma a direcionar ações para o cumprimento dos objetivos coletivos.

Dessa forma, ao responder as perguntas "para onde estamos caminhando? onde nos encontramos atualmente? e o que fazer para chegar lá?”, os gerentes das redes podem traçar ações estratégicas no curto, médio e longo prazo com diversas finalidades. Algumas dessas 
estratégias podem estar relacionadas com: (i) o mercado da rede, como a conquista de novos mercados e aumento da participação no setor; (ii) produto, como decisões relacionados ao portfólio de produtos e gestão de $\mathrm{P} \& \mathrm{D}$, sobretudo no que concerne a esforços, competências e recursos financeiros da rede; e (iii) tecnologia, com a capacidade de análise futura de tecnologias emergentes e transferências de tecnologias e conhecimentos entre os atores.

O TRM quando combinado às redes pode ser utilizado em contextos específicos como:

(i) Gestão das competências e recursos dos membros: o seu uso se dá, uma vez que existem diferenças entre atores da rede e, para desenvolvimento do TRM, é necessário que as disposições e as habilidades pessoais de cada ator sejam identificadas e trabalhadas para que a participação dos membros seja efetiva.

(ii) Gestão dos contatos estratégicos: o TRM pode ser abordado nas redes de contatos da própria rede e dos seus membros de modo a desenvolver laços fortes e fracos, conforme preconiza Granovetter (1973). Nesse sentido, é através de representações gráficas do TRM, que é possível se estabelecer quais contatos são estratégicos, de modo que se promova o fortalecimento do grupo, tanto pelo aumento da frequência e a confiabilidade dos contatos dentro e fora da rede (laços fortes), quanto pela estimulação da inovação, buscando acessar os contatos mais distantes da rede (laços fracos);

(iii) Auxilio às empresas líderes: visto que a configuração da rede pode apresentar um ator central, o TRM auxilia esse líder em conduzir as atividades da rede, possibilitando um roteiro estratégico a ser seguido pelos os outros diversos atores. Tal exposição pode ser observada na pesquisa de Muller-Seitz \& Sydow, (2012);

(iv) Relações com os stakeholders: no estabelecimento da TRM é possível inserir os objetivos e prioridades dos stakeholders (governo, empresas, sociedade e outras instituições) de forma a considerar as suas particularidades, entraves e interesses. Isso resulta no alinhamento dos objetivos dos interessados com a rede, direcionando os esforços com base na realidade dos envolvidos;

(v) Auxílio aos negócios: o TRM pode ser usado na gestão de riscos e outros gaps como recursos financeiros, busca por novos membros e parcerias, busca por recursos tangíveis e intangíveis, infraestrutura dentre outras atividades; e

(vi) Gestão da inovação: Como o TRM busca naturalmente o planejamento de novas tecnologias, é possível aplicá-lo no desenvolvimento e comercialização de novos produtos/serviços da rede, sobretudo nas redes o qual o aspecto tecnológico se faz preponderante, como as redes de empresas de base tecnológica.

Kappel (2001), ao contribuir com a proposição de taxionomias a TRM embasadas em diferentes tipos de abordagens, possibilitou uma alternativa de alinhar a própria ferramenta aos objetivos organizacionais, principalmente no que corresponde a mercado, produto e tecnologia. Assim, essas taxonomias são divididas em quatro áreas: roadmap de ciência/tecnologia, de indústria, de produto/tecnologia e de produtos. Essa divisão foi proposta conforme as variáveis de ênfase (trajetórias/posicionamento) e de propósito (expectativas e coordenação local). Torna-se importante salientar que roadmap é uma terminologia referente ao documento resultante do processo de roadmapping, ou seja, é a representação gráfica, o output do processo de TRM (Kappel, 2001). 
Desenvolveu-se, com base na proposta de Kappel (2001), uma adaptação à representação cartesiana do TRM com uma realidade do contexto de redes interorganizacionais (ver Figura 03).

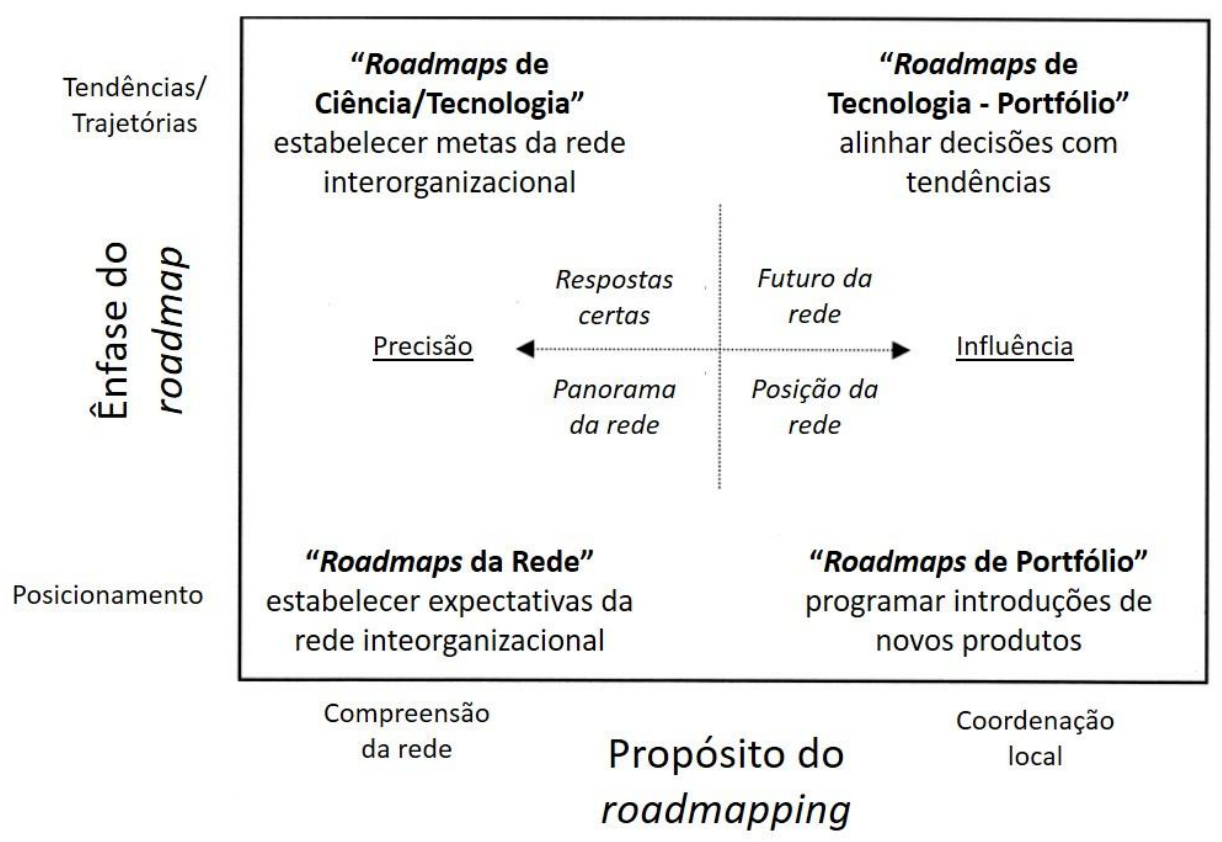

Figura 03: Taxionomia do roadmap

Fonte: Adaptado de Kappel, T. A. (2001). Perspectives on roadmaps: how organizations talk about the future. Journal of Product Innovation Management, 18(1), 39-50.

Assim, ao transpor para o contexto interorganizacional, os 'Roadmaps de Ciência/Tecnologia' podem ter como objetivo prover maiores entendimentos a respeito do futuro através da identificação de tendências específicas afim de resultá-las em metas para desenvolver o contexto das redes. Como exemplificação, esse tipo de adequação pode ser aplicado às redes incipientes buscando adentrar ou desenvolver mercados.

Os 'Roadmaps de Tecnologia-Portfólio' focam no alinhamento das tendências de mercado e tecnologia para o desenvolvimento do portfólio das redes. Pode-se aplicá-las às redes de empresas de alta tecnologia.

Já os 'Roadmaps da Rede' são decorrentes de um impulso técnico e do cenário competitivo que redes enfrentam. Assim, a necessidade e transferência de tecnologias, economia de escala e escopo, poder de barganha e demais aspectos quando associados ao contexto da rede, resulta neste tipo de roadmap. Como exemplo, esse tipo de roadmap pode ser melhor aplicadas às redes verticais, como cadeias produtivas.

Por fim, os 'Roadmaps de Portfólio' estão relacionados ao estabelecimento da direção e programação da evolução do portfólio das redes. Esse tipo de roadmap é mais indicado as redes de produtos customizados como redes moveleiras e têxtis.

Dessa forma, conforme a governança da rede vai estabelecendo seus objetivos estratégicos e prioridades, é possível que as mesmas desenvolva seus roteiros detalhadamente focando em alguma dessas áreas abordadas (Kappel, 2001).

No entanto, é importante frisar dois pontos importantes. O primeiro está relacionado a identificação da área foco do roadmapping considerando os aspectos do mercado, tendências 
de tecnologia e especificidades, e o segundo ponto refere-se à necessidade de customização da ferramenta, uma vez que a organização das redes de empresas podem ser diferentes quando comparadas umas às outras e podem mudar com o passar do tempo (Kappel, 2001).

Por fim, a complexidade da organização em redes como coordenação das atividades e membros, problemas de delimitação de fronteiras internas e externas, comprometimento e colaboração, dentre outros aspectos, fazem do TRM mais uma alternativa de gestão com importante função: é através do roadmap que a rede pode detectar as suas prioridades e oportunidades de maneira a envolver seus stakeholders pela estipulação de possíveis cenários para atuação durante o curto, médio e longo prazo (Kappel, 2001).

\section{Considerações Finais}

Retornando ao objetivo deste artigo de evidenciar a relação entre redes de empresa e technology roadmapping de forma a apresentar conciliações que facilitem e estimulem novos trabalhos no campo com foco em planejamento estratégico de redes interorganizacionais, é possível afirmar que, a partir da concepção de utilização de TRM para gestão de redes, a ferramenta apoia processos estratégicos das redes, a saber:

- Criação de portfólio de serviços e produtos;

- Transferência de tecnologia e de conhecimento;

- Integração de competências;

- Eficácia na utilização de recursos mediante metas e prazos;

- Aproximação do mercado;

- Reconhecimento de limites e entornos de redes (ambiente, stakeholders, atores, parceiros, etc.);

- Gestão de P\&D;

- Gestão de gaps;

- Identificação de tecnologias emergentes;

- Fortalecimento e desenvolvimento dos envolvidos.

Também foi apresentada possibilidade de adequação e utilização da ferramenta TRM no contexto de redes. Assim, após a governança da rede estabelecer seus objetivos e prioridades, cabe aos mesmos aplicar a ferramenta customizada (de acordo com a própria configuração da rede) em uma das áreas da taxionomia de Kappel (2001) adaptada ao contexto de redes afim de converter o planejamento estratégico em planos operacionais.

Neste sentido, o presente artigo atendeu ao objetivo proposto ao trazer contribuições teóricas como incentivo ao trabalho de governança de redes de empresas, o que resulta no seu destaque no mercado, melhoria de perspectivas futuras e orientação a ações gerenciais. Desta forma, destaca-se as contribuições deste trabalho à literatura uma vez que incentiva publicações nesse tema, reforçando o campo de estudos ainda pouco trabalhado, assim como contribui para as práticas gerenciais, possibilitando que gestores de redes possam vislumbrar uma gestão mais coesa e assertiva para as redes.

Cabe enfatizar também que, segundo estudos de Balestrin e Verschoore (2016) e Xavier Filho et al. (2015), as redes estabelecem seus objetivos com o intuito de concretizar seus ganhos competitivos, porém é também pela falta de objetivos contínuos e estratégias bem estabelecidas que as redes acabam por fracassar. Assim, a implementação do TRM na condução dos objetivos e na construção da estratégia da rede pode auxiliar que as redes alcancem seus ganhos competitivos. 
Apesar da contribuição exposta, a atual pesquisa apresenta limitações. A revisão de escopo foi desenvolvida através de uma única base de dados, o que limitou os resultados apresentados que poderiam ser em maior número e, portanto, com maiores dados a serem analisados, contribuindo para uma visão mais abrangente da área. Além desta primeira limitação, a partir dos artigos da amostra, o contexto das pesquisas foi puramente empíricas, porém, sem um intento maior de relação das temáticas. Por fim, cabe ressaltar que por se tratar de um artigo teórico se torna dificultosa a apresentação de pontos fortes e fracos da conciliação das temáticas, uma vez que tais aspectos são mais fáceis de serem observados em artigos empíricos.

Assim sendo, para trabalhos futuros, sugere-se a realização de mais pesquisas teóricas sobre a relação das duas temáticas. Outra sugestão é sobre aplicações empíricas que demonstrem mais claramente a utilização do TRM para contexto de redes, exemplificando benefícios, entraves, pontos fortes e fracos. As características estruturais de redes são altamente particulares e influenciarão o desenvolvimento do TRM e o formato do roadmap. Essas particularidades de desenvolvimento, se explicitadas, favorecerão a abrangência da literatura, dando continuidade ao objetivo de encorajar ações mais estratégicas e com maior visão para o futuro em redes.

\section{REFERÊNCIAS}

Alday, H. E. C. (2000). O planejamento estratégico dentro do conceito de administração estratégica. Revista FAE, Curitiba, 3(2), 9-16.

Arksey H \& O’Malley, L, (2005) Scoping studies: towards a methodological framework. International Journal of Social Research Methodology 8, 1-14.

Balestrin, A. \& Vargas, L. (2004). A dimensão estratégica das redes horizontais de PMEs: teorização e evidencias. Revista de Administração Contemporânea, 8(spe), 203-227.

Balestrin, J. A \& Verschoore, R. (2014). Réplica - Redes são Redes ou Redes são Organizações?. Revista de Administração Contemporânea, 18(4), 523-533.

Balestrin, J. A \& Verschoore, J. (2016). Redes de cooperação empresarial: estratégias de gestão na nova economia ( $2^{\mathrm{a}}$ ed.). Porto Alegre: Brookman. Contabilidade, Gestão e Governança, 16(3), 3-16.

Battistella, C., De Toni, A. F., \& Pillon, R. (2015). The Extended Map methodology: Technology roadmapping for SMES clusters. Journal of Engineering and Technology Management, 38, 1-23.

Bernal, M. L., Dornberger, U., Torres, M. O., \& Byrnes, M. T. (2009). Technology Roadmapping. International SEPT Program, Published by Universität Leipzig.

Bortolaso, I. V; Verschoore, J. R \& Antunes Junior, J. A. (2013). Práticas de Gestão de Redes de Cooperação Horizontal: Proposição de um Modelo de Análise, 16(3), 3-16. 
Capaldo, A. (2007). Network structure and innovation: the leverage of a dual network as a distincive relational capability. Strategic Management Journal, 28, 585-608.

Castells, M. (2005). A Sociedade em Redes. In Castells, M; Cardoso, G. A Sociedade em Redes: do conhecimento à Acção Política (17-30). Belém: Imprensa Nacional.

Castells, M. (2007). A Sociedade em Redes. In Castells, M. A era da informação: economia, sociedade e cultura (209-259), São Paulo: Paz e Terra.

Chiavenato, I. \& Sapiro, A. (2003). Planejamento estratégico. Elsevier. Brasil.

Fleury, S., \& Teixeira, S. M. F. (2011). Gestão de redes: a estratégia de regionalização da política de saúde. FGV Editora.

Fleury, S. (2014). As redes e a difusão de inovações. In: Conhecimento, inovação e comunicação em serviços de saúde. Editora Fiocruz.

Grandori, A \& Soda, G. (1995). Inter-firm network: antecedentes, mechanisms and forms. Organization studies, 16(2), 1-19.

Granovetter, M. S. (1973). The Strenght of Weak Ties. American Journal of Sociology, 78(1), 1360-1380.

Human, S. \& Provan, K. G. (1997). Na Emergent Theory of Structure and Outcomes in Small-Firms Strategic Manufacturing Networks. The Academy of Management Journal, 40(2), 368-403.

Johnson-Cramer, M. E.; Parise, S \& Cross, R.L. (2007) Managing chande throught networks and values. California Management Review, 47(3), 85-109.

Kappel, T. A. (2001). Perspectives on roadmaps: how organizations talk about the future. Journal of Product Innovation Management, 18(1), 39-50.

Lacoste, S. (2012). "Vertical Coopetition": the key account perspective. Industrial Marketing Management, 41, 649-658.

Lange, K., Müller-Seitz, G., Sydow, J., \& Windeler, A. (2013). Financing innovations in uncertain networks-Filling in roadmap gaps in the semiconductor industry. Research Policy, 42(3), 647-661.

Lee, S., \& Park, Y. (2005). Customization of technology roadmaps according to roadmapping purposes: Overall process and detailed modules. Technological Forecasting and Social Change, 72(5), 567-583.

Merofa, A \& Bueno, C. F. (2009, july) Coopetição: Uma Análise Teórica. Anais do IV Encontro de Estudos em Estratégia, Recife, PE, Brasil. 
Miles, R. E \& Snow, C. C. (1992). Causes of failure in network organizations. California Management Review, 34(2), 53-72.

Müller-Seitz, G., \& Sydow, J. (2012). Maneuvering between networks to lead-A longitudinal case study in the semiconductor industry. Long Range Planning, 45(2), 105-135.

Nohria, N. (1992). Is a network perspective a usefull way of studyng organizations?. In Nohria, N \& Eccles, R. G. Networks and organizations: structure, form and action (122). Boston: Harvard Business School Press.

Oliveira, A. L; Soares, A. S; Castro, C. C \& Mesquita, D. L. (2012). Redes de Inovação em Clusters: o local e o global na criação de vantagem competitiva. E-tech: Tecnhologias para Competitividade Industrial, 5(1), 1-15.

Phaal, R., Farrukh, C.J.P. and Probert, D.R. (2001), T-Plan: the faststart to technology roadmapping - planning your route to success, Institute for Manufacturing, University of Cambridge.

Phaal, R., Farrukh, C. J., \& Probert, D. R. (2004). Technology roadmapping-a planning framework for evolution and revolution. Technological forecasting and social change, 71(1), 5-26.

Phaal, R., Farrukh, C. J., \& Probert, D. R. (2005). Developing a technology roadmapping system. Technology Management: A Unifying Discipline for Melting the Boundaries, 31, 99-111.

Prahlad, C. K \& Ramaswamy, V. (2004). O Futuro da Competição: como desenvolver diferenciais inovadores em parcerias com clientes. Rio de Janeiro: Elsevier.

Rumrill, P. D., Fitzgerald, S. M. \& Merchant, W. R. (2010). Using Scoping Literature Reviews as a Means of Undestanding and Interpreting Existing Literature. Speaking of Research, 399-404.

Santos, M. D. M., Coelho, G. M., Santos, D. M. D., \& Fellows Filho, L. (2010). Prospecção de tecnologias de futuro: métodos, técnicas e abordagens. Parcerias estratégicas, 9(19), 189-230.

Xavier Filho, J. L. J., Paiva Júnior, F. G. D., Alves, S., \& Medeiros, J. J. (2015). Withdrawal of Cooperation in the Interorganizational Networks: Reflections Inspired by Weberian Social Action. RAM. Revista de Administração Mackenzie, 16(6), 159-189.

Zajac, E. J \& Oslen, C. P. (1993). From transaction cost to transaction value analysis: implications for the study of interorganizational strategies. Journal of Management Studies, 30 (1), 131-145. 